\title{
Les femmes constituent-elles un potentiel pour les entreprises ?
}

\author{
par Sophia Belghiti et Florence Rodhain
}

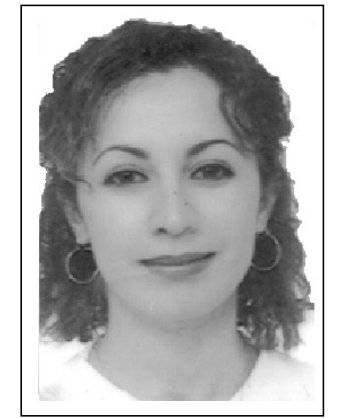

Sophia BELGHITI

Doctorante au CREGO-IAE Université de Montpellier II

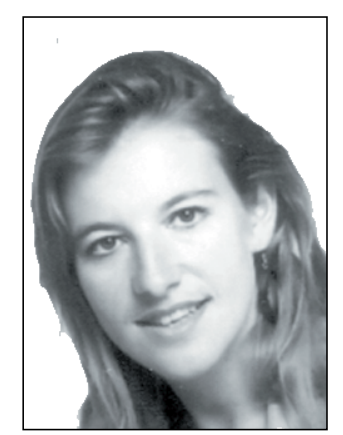

Florence RHODAIN Maître de conférence ISIM-CREGO Université de Montpellier II
«Etre femme aujourd'hui en France, c'est être majoritaire dans la population et vivre plus longtemps que les hommes. C'est être plus souvent diplômée que dans le passé, mais encore cantonnée dans une profession "féminine », plus engagée dans le monde du travail, mais plus souvent chômeuse, avoir une influence dans la vie sociale et politique en progrès, mais toujours loin de l'égalité avec les hommes » (Les Femmes. Portrait social. Collection Contours et Caractères, INSEE et Service des Droits des Femmes, février 1995).

Cela résume-t-il une réalité ? Incontestablement, oui.

Une chose est certaine : il n'est plus besoin de prouver que les femmes comme les hommes sont capables de faire des études au plus haut niveau. L'essor remarquable des scolarités féminines est un fait social majeur de ces dernières décennies. Elles sont majoritaires en terminales des lycées (54,8 \% en 1998-99) et réussissent au baccalauréat en plus forte proportion (au baccalauréat général 1998 , succès de $81,2 \%$ des filles et $76,5 \%$ des garçons; à l'ensemble des bacs technologiques, respectivement $83,3 \%$ et $75,7 \%$ ). Et elles sont aussi présentes que les garçons dans les études universitaires (56\% en 1997-98) (AFFDU, 1998). Tous les domaines d'études se féminisent, bien qu'à des rythmes très différents. Les formations professionnelles des femmes se sont améliorées et ne sont plus bornées à l'amélioration 
de leurs compétences dans la sphère privée (ménage, soins aux enfants...).

Cependant, si en droit elles ont accès à toutes les filières de formation et, maintenant, à toutes les professions, il n'en est pas de même dans les faits et dans la représentation en entreprise et sur le marché du travail...

L'abondance des plaidoyers sur la place des femmes dans la société d'aujourd'hui témoigne du regain d'intérêt qu'elle recommence à susciter. Cet intérêt ne précise pas si l'origine est un mouvement de fond ou tout simplement une réponse ponctuelle à l'ambiance paritariste du moment (loi constitutionnelle sur la parité hommes-femmes 1999) ? Là aussi, signalons le paradoxe français (ou l'exception française ?) : si la presse généraliste s'est emparée du sujet en multipliant articles, dossiers et émissions spécialisées, les chercheurs du champ le plus concerné par la forme actuelle de ce débat - les sciences de gestion - le boudent à quelques exceptions près.

Cela nous semble-t-il assez saisissant pour nous en préoccuper? Sûrement; ajoutons à que le gouvernement français se saisit lui-même du sujet en proposant, en mars 2000, une nouvelle loi sur l'égalité professionnelle, loi sur laquelle nous reviendrons aussi.

L'objet de cette étude est, dans un premier temps, de partir d'un phénomène constaté, qui est l'inégalité professionnelle entre hommes et femmes, et d'en repérer l'architecture. Autrement dit, nous commencerons par décrire la situation, nous exposerons ensuite les raisons qui nous semblent valider l'intérêt que les chercheurs auraient à s'intéresser à ce sujet, et nous terminerons en essayant de comprendre les raisons de leur indifférence actuelle.

Dans une seconde partie nous essaierons de concevoir le phénomène, traduit dans une certaine mesure, par la difficulté d'accès des femmes aux hauts postes de responsabilité ou ce qu'on appelle le «plafond de verre ».

Nous nous attacherons enfin, dans une dernière partie, à présenter quelques pistes de recherches sur ce thème.

\section{1 - L'égalité professionnelle entre hommes et femmes en France : entre mythe et réalité...}

\section{1 - Des chiffres pour soutenir un constat : l'égalité professionnelle en France entre hommes et femmes n'existe pas}

En France, les inégalités professionnelles entre hommes et femmes en France sont clairement mises en évidence dans les différents rapports produits par des instances nationales. Ces inégalités se rapportent principalement aux salaires, à la précarité et la probabilité d'être au chômage, et à la carrière (les chiffres qui suivent sont tirés d'études menées en 1998 et peuvent être consultés dans les références suivantes : Génisson, 1999, INSEE 1998, Ministère de l'emploi - 1, 1999 et Ministère de l'emploi - 2, 1999).

- Inégalité des salaires. En 1998, les femmes représentent environ $50 \%$ de la population active, et sont en moyenne plus diplômées que les hommes. Pourtant, les salaires perçus par elles sont en moyenne et tous emplois confondus inférieurs de $27 \%$ à ceux perçus par les hommes. Toutes choses égales par ailleurs, calcul assez délicat à réaliser, l'écart serait de l'ordre de 12 à $15 \%$. Parmi les salariés dont les salaires sont les plus faibles, on compte $85 \%$ de femmes, tandis qu'on ne retrouve que $27 \%$ de femmes parmi les salariés les mieux payés. Enfin, à diplôme égal, les femmes sont moins payées, et ce quelques soient les niveaux de diplôme considérés ;

- Inégalité face au chômage et à la précarité. En 1998, le taux de chômage touche $10,5 \%$ des hommes tandis qu'il touche $14 \%$ des femmes. L'INSEE a montré par ailleurs qu'être une femme - indépendamment d'autres caractéristiques (telles que le diplôme ou l'âge) - élève significativement la probabilité d'être au chômage. De plus, les femmes occupent plus souvent que les hommes, des emplois précaires ou à temps partiel. D'ordinaire, le 
temps partiel n'est pas choisi mais subi par les femmes. Alors que le temps partiel est assez marginal dans la population active masculine (de l'ordre de $5 \%$ ), il est important chez les femmes : environ un tiers d'entre elles. Or, d'après l'enquête menée en 1998 par l'INSEE, $40 \%$ des femmes occupant un travail à temps partiel souhaiteraient travailler davantage. Le travail à temps partiel n'est pas un problème lorsqu'il est choisi, mais peut conduire à des situations dramatiques lorsqu'il est subi, car il conduit à une moindre rémunération. C'est ainsi que le taux de femmes à bas salaire (c'est-à-dire moins de $5000 \mathrm{~F}$ ) chef de famille ou vivant avec un conjoint chômeur a augmenté pour atteindre, en 1998, $35 \%$ des situations. Enfin, $10 \%$ des femmes travaillant à temps partiel sont considérées comme vivant en dessous du seuil de pauvreté.

- Inégalité de carrière. Que ce soit dans le secteur privé ou public, les postes à haute responsabilité sont très peu occupés par les femmes. Elles ne représentent ainsi que $6 \%$ des cadres dirigeants dans les 5000 premières entreprises françaises. Si l'on observe la part des femmes dans la catégorie cadres et professions intellectuelles supérieures, elle s'élève à $34,2 \%$. Précisons que ce taux est gonflé par la sur-représentation du personnel féminin dans les activités d'enseignement, ce qui par conséquent, réduit la part des femmes dans les pures activités d'encadrement. La progression des femmes dans leur carrière est nettement moindre que celle des hommes :

- considérant les salariés ayant débuté comme « employé », $30 \%$ des hommes sont passés à la catégorie "profession intermédiaire », puis $26 \%$ d'entre eux à la catégorie « cadre supérieur » tandis que seules $17 \%$ des femmes sont passées à la catégorie «profession intermédiaire » et $4 \%$ d'entre elles à la catégorie « cadre supérieur »;

- considérant les salariés ayant débuté en tant que «profession intermédiaire », $38 \%$ des hommes contre $17 \%$ de femmes sont devenus cadres ;

force est de constater - à la lumière de ce descriptif de la situation française en 1998 - que l'égalité profes- sionnelle entre hommes et femmes n'est pas encore une réalité, même si des progrès ont été réalisés au cours des dernières décennies (c'est ainsi par exemple qu'en dix années la proportion des femmes dans la catégorie cadres et professions intellectuelles supérieures a progressé de $27 \%$ ).

\section{2 - Au-delà du constat, la question de l'égalité professionnelle présente de nombreux intérêts micro et macro économiques}

«L'admission des femmes à l'égalité professionnelle serait la marque la plus sûre de la civilisation, et elle doublerait les forces intellectuelles du genre humain ». $\mathrm{Si}$, contrairement à cette affirmation de Stendhal on se place d'un point de vue strictement économique, il apparaît que l'égalité professionnelle entre hommes et femmes participe au développement du pays ainsi qu'à celui de l'entreprise. Cette égalité pourrait même être utilisée comme avantage compétitif pour les pays ou les entreprises les plus avancées dans le domaine. Les deux niveaux d'analyse doivent être séparés pour être mieux cernés.

- Niveau macro-économique. Un rapport du Conseil d'analyse économique (1999) montre que les femmes sont loin de «prendre le travail des hommes » mais au contraire, qu'elles participent au développement du pays. Les études comparatives internationales démontrent une corrélation entre un taux d'activité féminine élevé et un fort taux de croissance économique du pays, ainsi qu'un taux de chômage structurel faible. D'un point de vue compétitif, on peut considérer que les pays s'engageant dans la voie de l'égalité professionnelle possèdent une arme compétitive contre les pays s'y engageant à reculons. C'est en tout cas l'argument proposé par Schwartz (1992) qui suggère que les USA possèdent un avantage compétitif sur un pays comme le Japon où les femmes sont absentes des équipes dirigeantes. 
- Niveau micro-économique. L'égalité hommes-femmes profite également à l'organisation :

- l'arrivée des femmes à tous les niveaux de l'entreprise peut être une occasion de réexamen des modes de fonctionnement (Génisson 1999) en vue d'une amélioration de ceux-ci ;

- l'entreprise qui traite de manière parfaitement égale ses salariés a l'opportunité d'améliorer son image, en particulier en direction de ses actionnaires, surtout lorsque ceux-ci sont pour une bonne part constitués de femmes (Schwartz, 1992) ;

- lorsque les femmes quittent l'organisation parce qu'elles n'y trouvent pas le soutien dont elles ont besoin dans leur carrière, l'entreprise doit faire face à des coûts de recrutement et de formation (Schwartz, 1992);

- lorsque l'entreprise n'offre pas l'équité entre hommes et femmes, elle risque de perdre en productivité et en efficience. En effet, si les femmes concentrées aux bas niveaux de la pyramide doivent rendre compte de leur travail à des hommes peu ou pas forcément mieux qualifiés qu'elles, le talent et la vision de ces employées risquent d'être étouffés par les limitations de leurs supérieurs hiérarchiques (Schwartz, 1992).

\section{3 - Pourtant le problème est très peu considéré par le chercheur en sciences de gestion...}

Si le sujet de l'égalité entre hommes et femmes dans les organisations, suscite de nombreuses recherches aux Etats-Unis dans le domaine de la gestion et ce, depuis plus de vingt ans, on constate à l'inverse un quasi silence sur la question en France. Ce silence pourrait s'expliquer par deux phénomènes conjoints : un manque d'informations sur la question de l'égalité, et une absence de revendications de la part des femmes.

- Une absence d'informations. Cette absence d'informations s'explique d'une part, par le manque d'études et de statistiques nationales sur la question et, d'autre part, par le manquement des entreprises qui ne remplissent pas ou très mal leur obligation de rapport annuel sur la situation comparée hommes-femmes (Genisson, 1999).

- L'absence d'informations au niveau macro-économique. Les sources statistiques ne sont pas toujours sexuées, et lorsqu'elles le sont, leur exploitation n'est pas toujours de mise, faute de demande en ce sens (Génisson, 1999). Ce n'est que récemment, que des travaux de grande envergure ont été entrepris pour analyser la situation professionnelle des femmes, et ce sous l'impulsion de l'Union Européenne et de la France. Ces travaux sont presque toujours élaborés par des femmes (voir l'ouvrage collectif de Margaret Maruani ou le rapport du Conseil d'analyse économique présenté par Béatrice Majnoni d'Intignano ou encore le rapport remis au Premier ministre par Catherine Génisson).

- L'absence d'informations au niveau micro-économique. Deux lois auraient dû permettre de pallier les insuffisances d'informations sur la question de l'égalité professionnelle dans les entreprises. Premièrement, les lois « Auroux » qui s'adressaient aux branches professionnelles. Celles-ci sont dans l'obligation, d'après cette loi, de dresser annuellement une situation comparée entre les hommes et les femmes dans la branche concernée. On constate que ce bilan est dans le meilleur des cas, élaboré de façon trop succincte et, généralement, inexistant (Génisson, 1999). Deuxièmement, la loi « Roudy » contraint toute entreprise française de plus de 50 salariés à produire un rapport annuel sur la situation comparée entre les sexes. Or, moins de la moitié des entreprises françaises produisent ce rapport (Génisson, 1999). Quant aux entreprises qui le produisent, elles ne le font pas dans « l'esprit de la loi ». En effet, le rapport est censé servir de base à des discussions et des négociations dans l'entreprise avec les salariés. Cependant, les entreprises interrogées lors de la constitution du rapport demandé par le Premier ministre (Génisson, 1999) estiment qu'il n'est pas du tout utilisé par l'entreprise pour la discussion. Il ne représente tout au plus qu'une 
photographie, sans analyse en vue d'une amélioration de la situation.

- Une absence de revendications. On constate une absence de revendications de la part des femmes sur l'égalité professionnelle. Cette absence peut s'expliquer par deux raisons principales : premièrement, du fait de l'absence des femmes aux postes qui leur permettraient de faire valoir leur vision et deuxièmement, du fait d'un sentiment de satisfaction de leur part, comparable à celui des hommes alors que leurs situations sont inégales.

- Absence des femmes aux postes permettant de faire valoir leur vision. Les femmes sont encore très clairement minoritaires à la tête des entreprises, dans les syndicats, en politique ainsi que dans la haute fonction publique. Elles sont donc sous-représentées aux postes permettant de prendre en compte leurs désidérata, ce qui explique en partie l'absence de revendications sur le sujet en France.

- Des satisfactions comparables en dépit de situations inégales. Un document passionnant élaboré par le ministère de l'emploi et de la solidarité (1999), au titre évocateur «Hommes et femmes : des satisfactions comparables en dépit de situations inégales », montre que, si, face au travail, les hommes et les femmes sont inégaux, les satisfactions sont quant à elles étonnamment proches. Ce niveau de satisfaction égal à situation inégale explique aussi en partie le manque de revendication de la part des femmes sur l'égalité professionnelle. Les jugements portés sur les salaires n'offrent, dans l'enquête réalisée par le ministère, aucune différence significative, alors que ces salaires sont significativement différents. Est-ce que les femmes, comme le suggère le document du ministère du travail, auraient « entièrement intériorisé et accepté les inégalités de salaire agissant à leur encontre » ? Il faut tenir compte du fait qu'apparemment le jugement des femmes est « moins mécaniquement déterminé par le montant du salaire », toujours d'après la même étude. Ce qui explique en partie la satisfaction égale. Autre élément d'explication intéressant : les femmes auraient tendance à se comparer non pas aux hommes, mais à leurs mères.
Lorsque c'est le cas elles ne peuvent être que satisfaites de leur situation. Le simple fait de travailler constitue souvent un progrès par rapport à la situation de leurs mères. Comme le souligne le rapport du ministère « la généralisation du travail féminin n'a pas réussi à émousser la vigueur de ce sentiment positif ».

\section{2 - Inégalité professionnelle entre hommes et femmes, comment l'expliquent les théories : cas des femmes cadres}

Même si elles sont de plus en plus nombreuses à accéder au statut de cadre, les femmes demeurent minoritaires dans les organigrammes de décision. Rappelons-le, sur les 26700 dirigeants recensés dans les états-majors, seules 1683 (soit $6 \%$ ) sont des femmes (5 000 Entreprises Leaders en France, Nouvel Economiste, 1998).

Le chemin d'accès aux postes de direction n'est donc pas tout tracé pour les femmes.

Deux réactions face à ce constat : ou bien on considère que le phénomène est naturel et on ne se préoccupe pas de lui trouver de causes ou d'explications; ou bien on le considère comme étant "incongru » et l'on tente de l'expliquer. Notre objet n'est pas de remonter à la genèse de l'homme pour y trouver les justifications à «la domination masculine » (Bourdieu, 1998), mais tout simplement de passer en revue les explications qui ont été avancées, afin d'établir que la communauté des chercheurs (toutes disciplines confondues), ne considèrent pas que le phénomène soit "dans l'ordre des choses », mais tentent bien, à défaut d'y remédier, de l'expliciter.

Nous commencerons par des explications issues $d u$ champ économique voisin, nous discuterons ensuite de celles issues du champ de la sociologie et nous terminerons par celles que nous considérons attachées au champ gestionnaire. 


\section{1 - Interprétations d'explications empruntées aux théories économiques}

Les explications retrouvées dans les théories économiques concernent la ségrégation professionnelle hommes-femmes. Or, les études qui traitent de ce sujet s'intéressent, en fait, non au phénomène lui-même, mais à ses répercussions sur les rémunérations comparées des hommes et des femmes (Anker, 1997). Nous considérons de la même façon, que les difficultés d'accès des femmes aux postes de haut niveau, qui ont des causes diverses, peuvent extraire les répercussions de la ségrégation professionnelle selon le sexe.

Pour expliquer le phénomène, les modèles économiques s'emploient à justifier les raisons pour lesquelles les femmes préfèrent certains postes à d'autres, et pourquoi les employeurs préfèrent recruter soit des hommes, soit des femmes pour certains emplois. Et ce, sous les hypothèses néoclassiques de rationalité des acteurs et d'efficacité du marché : les salariés recherchent les emplois qui maximisent leurs rémunérations et les employeurs sont en quête d'employés qui réduisent leurs coûts et maximisent la productivité de leur affaire. Les modèles économiques considèrent que les femmes, les hommes et les groupes minoritaires forment des ressources différentes sur le Marché du Travail. Deux théories sont utilisées pour expliquer le phénomène de ségrégation professionnelle (Anker, 1997), celle du capital humain et celle de la segmentation du marché du travail.

Dans l'optique néoclassique du capital humain, le personnel féminin est :

1- de niveau inférieur en ce qui concerne les capacités qu'il apporte sur le marché du travail (formation des femmes moins poussée que celles des hommes), et les capacités qu'il est apte à acquérir une fois qu'il travaille (à cause des interruptions de l'activité professionnelle qu'entraînent les naissances, les responsabilités familiales...).
2- considéré comme plus coûteux que le personnel masculin, à cause de l'existence supposée de certains coûts indirects (coûts d'installations à aménager : toilettes, crèches pour les enfants, etc..). 3- plus souvent absent que le personnel masculin, plus souvent en retard (à cause des responsabilités familiales). Aussi le turnover des femmes serait donc plus élevé que celui des hommes, et donc plus coûteux.

Quant à la théorie de la segmentation du marché du travail, elle distingue deux marchés : le secteur primaire et le secteur secondaire (Doeringer et Piore, 1971), les emplois statiques et les emplois dynamiques (Standing, 1989), le secteur formel et le secteur informel (structuré et non structuré) (BIT, 1975). Ainsi, les conditions de recrutement, de rémunération ou de promotion sont variables d'un secteur à l' autre, le secteur primaire étant considéré comme offrant les meilleures conditions.

Dans le premier secteur, la gamme de professions est élargie et les rémunérations élevées, et cela devrait justifier le recrutement par les entreprises d'éléments les plus qualifiés, les hommes en l'occurrence, possédant une formation et une expérience plus développées.

Dans le second secteur, les entreprises font face à une concurrence accrue qui les pousse à accorder des rémunérations comparativement faibles. On comprendrait ainsi, pourquoi il y a concentration des femmes dans un petit nombre de professions dans ce secteur (Bergmann, 1974).

Ces analyses, issues des théories économiques afin d'expliquer la situation des femmes, semblent incomplètes pour comprendre pourquoi ces dernières n'accéderaient pas aux mêmes postes que les hommes, alors qu'elles possèdent les mêmes formations et compétences qu'eux.

Le potentiel de salaire et d'opportunité d'avancement augmentent certes, avec le niveau de formation atteint, mais les hommes gagnent toujours plus que les femmes et montent plus facilement dans la hiérarchie, et ce, à 
niveau de formation et même de responsabilité similaires. Cette hiérarchie de privilèges économiques contredit l'équation du capital humain. D'autre part, en ce qui concerne le turnover, Janet Chafetz (in Ollenburger and Moore, 1992) révéla dans son étude de 1976 sur les employeurs et les employés hommes et femmes, que les taux de turnover étaient équivalents pour les hommes et les femmes dans des emplois similaires. Ce qui distingue les deux groupes ce sont les raisons du turnover. Les hommes quittent leurs emplois pour ceux qui leur procurent de plus grandes opportunités promotionnelles. Les femmes quittent leurs emplois pour s'occuper de leurs enfants. Le résultat pour les employeurs est le même : ils perdent le capital formation investi dans leurs salariés. Le résultat pour les travailleurs est un peu différent, si l'on considère que les hommes choisissent les postes qui augmentent leur chance de promotion et exploitent leur ancienneté ; et que les femmes quittent le marché du travail, perdent de l'ancienneté et perturbent le cours des promotions (Ollenburger \& Moore, 1992).

D'autre part, l'activité professionnelle des femmes est de plus en plus continue, leur formation est de plus en plus égale à celle des hommes, et le poids des tâches ménagères et familiales se réduit. Il en résulte que l'expérience professionnelle des femmes devrait les amener aux même aspirations et postes que les hommes. Or ce n'est pas encore le cas.

En fait, les éléments qui peuvent justifier la part importante de la charge familiale assumée par les femmes, ou par exemple pourquoi les organisations développent des stéréotypes sur leurs compétences, se situent hors du champ de l'emploi, et quelque part hors de la compétence économique. Ces facteurs ou comportements freinant l'ascension des femmes justifient le recours aux théories socioculturelles et gestionnaires pour approfondir les explications. Le pragmatisme des sciences de gestion peut en effet aller au-delà des limites des explications « économistes », basées sur des hypothèses de rationalité étendue, en analysant avec plus de discernement les apports des interactions individu-organisation.

\section{2 - Explications du champ sociologique : les analyses socioculturelles}

Nous partons de l'idée que, d'une façon ou d'une autre, les restrictions culturelles pesant sur les femmes en société se traduisent inévitablement dans l'entreprise. Pour plusieurs théoriciens des organisations, le comportement des organisations suit de près celui du rapport social. Aussi, le régime politique et social global reposet-il d'une certaine manière sur le système productif dominant dans l'entreprise. «Les organisations répondent aux finalités sociales et, à ce titre, on observe à l'intérieur de celles-ci, les mêmes configurations que celles qui sont à l'œuvre dans les efforts de transformation sociale actuels» (Coutrot, 1996).

En effet, si l'on considère les domaines publics et privés dans leur interaction, on retrouve indéniablement des liens : la façon dont chaque société organise les rôles et les relations entre les deux sexes a de profondes répercussions sur l'organisation de toute la société. C'est manifeste, par exemple en ce qui concerne la structuration de la famille (Eisler, 1995) : dans les sociétés patriarcales, là où le rôle des hommes et des femmes est strictement délimité, le modèle familial est en général très hiérarchisé et autoritaire. Dans les sociétés moins masculines, dans l'attribution des rôles, le modèle familial est souvent moins rigide. La manière dont la société organise les rapports entre hommes et femmes dépend de la structuration des rôles à l'intérieur de la famille et peut avoir autant d'implications socio-économiques que sur la vie personnelle des individus.

Ceci nous conduit à considérer le fameux « effet de miroir » et à avancer que la situation des femmes et leur avancement dans la hiérarchie des entreprises sont étroitement liés à la réalité socioculturelle à laquelle elles sont confrontées. Leur difficulté d'accès à des postes de haut niveau est associée, entre autres, à l'état des mentalités et des représentations dominantes sur le partage des responsabilités familiales et des tâches domestiques 
entre hommes et femmes. Cet état de mentalité est ainsi le reflet, plus ou moins prononcé, de la structuration de la société, de la famille et de la culture.

Nous rejoindrons dans cette optique le propos de Duchéneaut (1999) suggérant que les barrières rencontrées par les femmes managers sont les résultantes de cinq forces parmi lesquelles : le degré de féminité ou de masculinité de la socioculture nationale.

L'étude la plus classique sur ce plan a été réalisée par Geert Hofstede (1987), qui définit quatre dimensions de différences culturelles pouvant être comparées d'un pays à l'autre : la distance hiérarchique, le degré d'individualisme, le degré de masculinité et le contrôle de l'incertitude.

L'indice de masculinité et son complément, l'indice de féminité, sont situés sur une échelle de 0 à 100 . Les conséquences de la dimension de la masculinité en matière d'organisation du travail font apparaître peu de ségrégation dans les métiers dans les « pays à culture féminine » (Hofstede et Bollinger, 1987). A priori, les pays à indice de masculinité fort dressent vraisemblablement davantage de barrières à l'entrée et freinent davantage la progression des femmes dans les postes à responsabilité que les pays à indice de féminité élevé (Duchéneaut 1999). La Suède, pays ayant la culture la plus féminine sur l'échelle de Hofstede, a un taux de postes managériaux et d'administration de $39 \%$ pour les femmes, alors que le Japon, avec sa culture masculine la plus élevée, présente un taux de $8 \%$ (Nations Unies, PNUD, 1995).

Ceci nous amène à ouvrir une parenthèse pour évoquer la parité, le principe politique qui a suscité tant de débats, défini d'ailleurs, plus comme un accélérateur de conscience qu'un principe d'égalité (Bachelot et Fraisse, 1999). Les « antiparitaristes » telle Elisabeth Badinter soulignent qu'il faut commencer par résoudre le problème de la parité domestique et celui de la parité professionnelle, et qu'ensuite, tout naturellement, la parité politique suivra. Les défenseurs de la parité pensent que si, dans la classe politique, la femme est à parité avec l'homme, cela aura un effet d'entraînement sur l'ensemble du corps social. Encore l'illustre effet de miroir, entre le pouvoir politique et la société. Si dans les lieux emblématiques de pouvoir il n'y avait pas de femmes, les entreprises hésiteraient inconsciemment à accorder à des femmes des fonctions de direction, de pouvoir. En tout cas, sur le plan symbolique, l'image de la politique et du pouvoir, dominée par les hommes, renforce peut-être inconsciemment l'idée que les femmes ne sont pas faites pour accéder aux hautes fonctions. La question n'est pas si simple, rappelons encore que la Suède, qui détient le record du monde de la représentation parlementaire des femmes (40\% en 95), a été désignée en 1994 par les Nations-Unis comme le pays où les femmes vivent le mieux. Ajoutons toutefois que les postes de management supérieur occupés par les femmes sont de $7 \%$ en France, $3 \%$ en Norvège et $2 \%$ au Danemark (Adler, 1993). Ils sont relativement faibles, alors que l'on pourrait s'attendre à ce qu'ils soient supérieurs dans les pays nordiques. Ceci nous détermine à constater que l'effet de miroir est, bien sûr, un effet réflexif et même dual : politique/société, société/entreprise, mais la relation n'est pas étendue à une relation transitive au sens algébrique du terme, d'où l'on pourrait déduire dès lors, la relation politique/entreprise, du moins, on ne peut pas encore l'affirmer... Nous voyons plutôt la relation dans un schéma triangulaire, où les trois angles du polygone seraient occupés par les trois domaines : politique, société et entreprise.

\section{3 - Les femmes cadres et le «plafond de verre »: explications au niveau de l'organisation}

Dans les années soixante-dix, lorsqu'il y avait peu de femmes dans la sphère des hauts niveaux de direction, on expliquait leur absence par le fait qu'elles ne possédaient pas les compétences appropriées à ces postes.

Aux Etats-Unis, à partir des années quatre vingt, un terme est créé pour désigner les barrières invisibles pour la promotion des femmes dans les niveaux supérieurs de la hiérarchie : le «glass ceiling » traduit en français par le « plafond de verre ». Les barrières qui constituent le plafond de verre étant générées par des préjugés d'ordre 
comportemental et organisationnel empêcha les femmes d'accéder aux plus hautes responsabilités. C'est ainsi que la «Federal Glass Ceiling Commission » a été créée en 1991 afin d'identifier, au niveau des organisations, les barrières et les préjugés spécifiques, dans leur culture ou environnement professionnel qui bloquent l'avancement des femmes et des minorités.

Le « plafond de verre » existe bien, et l'on cite parmi les barrières qui le constituent le fait que « les femmes sont coupées des réseaux, aussi bien formels qu'informels, dont l'appui est essentiel pour obtenir de l'avancement au sein de l'entreprise ». Alors que les hommes se constituent des réseaux en dehors de leurs heures de travail, les femmes préfèrent - et sont bien obligées de consacrer leur temps libre à la vie familiale. Les responsabilités que les femmes assument en matière d'éducation des enfants et de tâches ménagères pèsent également sur leur carrière (cf. Recherches liées au WFC, partie 3.2).

Les barrières rencontrées par les femmes dans leur progression ont plusieurs origines et des combinaisons diverses. Le secteur d'activité de l'entreprise, le niveau de responsabilité exercé et la plus ou moins forte masculinisation des stéréotypes managériaux, en font partie, et peuvent ainsi freiner ce processus de progression (Duchéneaut, 1999).

C'est ainsi que dans les pays anglo-saxons, plusieurs recherches et études tentent d'expliquer et d'analyser les mécanismes intra-organisationnels responsables de cette situation. Car rappelons-le, la plupart des études faites sur les femmes cadres ces vingts dernières années restent d'essence nord-américaine. On en retiendra la difficulté des femmes à briser le « plafond de verre » et le fait que l'univers des entreprises demeure essentiellement masculin et reflète une réalité proche des valeurs sociales et culturelles dominantes. Les décisions de recrutement ou promotion reposent sur des dominations diverses et floues qui peuvent favoriser des interprétations pouvant aller dans le sens des exigences tacitement « masculines ».

Dans ce sens, l'entreprise, à l'instar de l'école pour Bourdieu, peut être présentée comme une institution reproduisant les rapports sociaux de domination. Cette reproduction s'effectue par la généralisation d'un système de « valeurs », idéaux collectifs qui définissent dans l'entreprise les compétences désirables, puis leur mode de traitement à travers des dispositifs de gestion et d'organisation...

C'est ainsi que les femmes ayant réussi disent souvent avoir consciemment adopté un style « masculin », afin d'entrer dans le moule des valeurs attendues par leurs collègues masculins.

En effet, les recherches montrent que les femmes qui accèdent à des postes de "senior management", ont généralement des spécificités communes et disent souvent qu'elles ont (Ragins, Towsend \& Mattis, 1998), certes développé un style convenant aux hommes, mais également :

- «Dépassé les attentes de l'entreprise », en étant irréprochables, en quelque sorte « superperformantes », en fournissant de meilleurs résultats que leurs homologues masculins.

- « Recherché des responsabilités difficiles ou à forte visibilité »: missions difficiles délaissées souvent par les autres, par crainte de l'échec.

- «Eu un mentor influent », un parrain qui les a aidées et soutenues (cf. Recherches liées au Mentoring, partie 3.1).

Les travaux nord-américains sur le «plafond de verre » sont abondants, et les explications sur l'inégalité de carrière entre hommes et femmes sont nombreuses et variées. Toutefois, trois perspectives peuvent être présentées comme majeures dans ce thème d'études (Parker \& Fagenson, in Burke and Davidson, 1994) :

La première approche pour comprendre l'inégalité professionnelle entre homme et femmes est "gendercentered ", approche centrée sur les différences entre hommes et femmes (Horner, 1972; Terborg, 1977, Riger and Galligan, 1980). L'argument basique de cette approche est que le sexe, que ce soit à cause des origines biologiques ou des influences sociales, détermine la plupart des aptitudes, compétences et autres caractéris- 
tiques, expliquant les comportements. Dans cette approche, on peut distinguer trois visions différentes :

- la première stipule que les femmes possèdent moins de compétences et d'aptitudes nécessaires au management que leurs collègues masculins. Les recherches montrent que cette perspective est encore présente dans les esprits de nombreux managers masculins aujourd'hui (Brennan et al., 1989, Heilman et al., 1989) ;

- la seconde, au contraire, soutient que les femmes possèdent des compétences différentes et même meilleures que les hommes ;

- la troisième est celle appelée « approche des contributions complémentaires » (Adler \& Izraeli, 1988). Les différences sont certes basées sur le sexe, mais cette théorie ne suggère pas que les femmes ou les hommes soient fondamentalement meilleurs managers, mais peuvent être respectivement meilleurs dans certaines tâches managériales.

La seconde approche soutient que les différences observées et observables dans le comportement managérial des hommes et des femmes sont provoquées par des situations différentes sur le lieu du travail. Cette perspective appelée l'approche par la structure ou la situation organisationnelle (the situation or organizational structure approach), affirme que les différences avec lesquelles les femmes managent au travail, pourraient être dues aux caractéristiques de l'organisation. Les caractéristiques de personnalités - ou les comportements qui sont l'expression de la personnalité - peuvent changer en fonction de la situation (Freeman, 1990).

La troisième approche, intitulée "gender-organizationsystem »(GOS), a été utilisée pour expliquer la diversité de style de management. Elle adopte les aspects des deux approches précédentes. En effet, elle reconnaît qu'hommes et femmes sont différents et ont donc des comportements différents, mais que les situations organisationnelles influencent également les comportements des individus (Fagenson, 1990, 1993). Dans cette approche, l'individu et son organisation ne peuvent être compris séparément de la société (et culture) englobante. En outre, lorsque l'individu, l'organisation, ou le système dans lequel ils sont enracinés, changent, les autres composantes changent aussi (Fagenson, 1993). Cette théorie fournit un cadre moins réducteur pour l'analyse des organisations, parce qu'elle considère les statuts des hommes et des femmes dans les organisations, en tenant compte du contexte organisationnel et socioculturel qui fait émerger les différences ou les égalités. Au lieu d'affirmer que les femmes sont meilleures que les hommes ou que les hommes sont meilleurs que les femmes, propos de l'approche centrée sur le genre, ou que les organisations ont particulièrement besoin de changer, ce qui est l'argument central de l'approche organisationnelle, l'approche GOS suggère que les gens, les organisations, les rôles et les sociétés, changent simultanément en réponse aux transformations environnementales, bien qu'à des rythmes différents. Le fait est qu'il existe des rythmes et des vitesses différents qui pourraient expliquer pourquoi le progrès envers l'égalité managériale en faveur des femmes est différent à travers le monde.

C'est cette approche que nous trouvons la plus pertinente car la moins réductrice. Nous pensons que la situation des femmes, leurs difficultés d'avancement ou les attitudes face à leur carrière, dépendent et sont étroitement liées au contexte socioculturel et même institutionnel dans lequel elles évoluent. Certes et avant tout c'est un problème d'organisation, cependant; la manière, avec laquelle il peut être appréhendé en France n'est pas la même que celle avec laquelle il peut l'être aux Etats-Unis (où le féminisme est plus important qu'en France).

\section{Difficultés d'accès des femmes aux hauts postes de management, quelques voies de recherche}

De nombreuses voies de recherche peuvent être citées autour du problème de la difficulté d'accès des femmes à des postes de direction. Nous en citerons trois : le mentoring, les conflits entre la vie de famille et le 
travail, et enfin, la place du droit dans le problème de l'égalité professionnelle.

\section{1 - Les recherches liées au mentoring}

Le mentoring, terme anglophone signifiant parrainage, est de plus en plus utilisé par les entreprises françaises. Il est amusant de souligner que les Américains utilisent le terme «protégé » ou «protégée » (avec les accents) alors que par exemple en France, à General Electric, on utilise le terme « menty » pour signifier « protégée » (alors que le terme « menty » n'est pas utilisé aux EtatsUnis). Le mentor est un parrain expérimenté, généralement plus âgé que son protégé, qui consent à épauler un jeune salarié dans l'organisation afin de faciliter son développement personnel (Hunt et Machael, 1983 ; Kram, 1985 ; Noe, 1988 ; Ragins et Scandura, 1994). Le mentoring doit profiter aussi bien aux individus qu'à l'organisation. En général, le protégé est un jeune professionnel caractérisé par de fortes aspirations à faire carrière (Hunt et Michael, 1983). La relation mentorprotégé est habituellement informelle. Cependant et de plus en plus souvent, on a vu se créer au sein des organisations anglo-saxones des programmes de mentoring (Roche, 1979 ; Klauss, 1981 ; Noe, 1988). Ces entreprises développent en leur sein une « culture mentoring » (Murray et Owen, 1991 ; Kram et Bragar, 1992 ; Parker et Kram, 1993). Les chercheurs ont montré que le mentoring est corrélé à des revenus plus importants (Dreher et Ash, 1990), à la mobilité dans la carrière (Scandura, 1992), à la satisfaction dans la carrière (Fagenson, 1989), à la satisfaction quant au salaire perçu (Dreher et Ash, 1990) et enfin, à la promotion (Dreher et Ash, 1990 ; Whitely et al., 1991). Cette dernière relation amène à poser la question de l'intérêt du mentoring pour les cadres féminins. Dans quelle mesure le mentoring permettrait-il de promouvoir les femmes dans les organisations ? Voici une idée de recherche aux intérêts pratiques facilement identifiables. Si le mentoring accélère effectivement la promotion des cadres féminins (Ragins, Towsend \& Mattis, 1998) soulignent à cet égard que certaines femmes citent la présence d'un mentor comme facteur de succès à la promotion se pose alors la question comment favoriser cette pratique au sein des organisations désireuses de former et promouvoir leurs cadres féminins. Par exemple, est-il plus efficace de créer des programmes formels de mentoring ? Ou bien est-il préférable d'impulser une « culture mentoring » dans l'organisation, sans que celle-ci prenne une forme bien précise?

\subsection{Les recherches liées au Work- Family Conflict}

Le concept de "Work-Family Conflict» (WFC) peut se définir comme "une forme de conflit de rôle chez la personne carriériste, apparaissant lorsque les exigences du travail et les exigences de la famille sont mutuellement incompatibles » (Higgins and Duxbury, 1992). Les modèles de WFC stipulent que le conflit apparaît lorsque les demandes de participation dans un domaine de la vie sont incompatibles avec la demande de participation dans un autre domaine; ces conflits peuvent avoir un effet important, à la fois sur la qualité de la vie de famille et sur la qualité de vie sur le lieu de travail (Greenhaus, 1988 ; Greenhaus and Beutell, 1985 ; Netemeyer et al., 1996). Etant donné que les femmes sont traditionnellement plus attachées que les hommes aux tâches familiales, on peut supposer qu'elles sont plus exposées au WFC. Il n'est pas rare que le début de la carrière d'une femme coüncide avec la période de la vie où elle entame une vie familiale. A cette période, les femmes doivent gérer une carrière, une maison et éventuellement, des enfants. Les femmes ayant des vies familiales pleines peuvent être perçues comme manquant d'un engagement fort dans leur travail ou comme n'étant pas aussi sérieuses dans leur carrière que leurs collègues masculins (Barinaga, 1992). Des recherches signalent que c'est à partir de ce moment que les femmes commencent à prendre du retard par rapport aux hommes en terme de promotion. Duxbury et Mills (1990) ont analysé 359 couples, où les conjoints se définissent tous deux comme carriéristes. Ils ont trouvé que les femmes plaçant 
leur carrière avant celle de leur mari ou à égalité avec lui, subissent plus de conflits dans leur vie privée que les femmes plaçant leur carrière en seconde position. Autre résultat intéressant : les femmes ramenant du travail à faire à la maison doivent faire face à de plus nombreux conflits familiaux que les hommes agissant de même. Comme le soulève Schwartz (1992), un faible pourcentage d'hommes et de femmes privilégient particulièrement leur carrière au détriment de leur famille. De la même façon, un très faible pourcentage d'hommes et de femmes sont totalement centrés sur la famille. Mais la grande majorité d'hommes et de femmes souhaitent combiner harmonieusement famille et carrière et souhaitent de temps à autre, pouvoir passer d'un centre d'attention à l'autre, et ce tout le long de leur vie. Entreprendre des recherches sur cette question du Work-Family Conflict permettant de mieux comprendre le phénomène pourrait déboucher sur des recommandations pour la gestion des ressources humaines. Préconiser la pratique de la flexibilité pourrait être un exemple de recommandation. Adopter plus de flexibilité dans les horaires de travail, laisser les salariés travailler à la maison, ne permettrait-il pas aux cadres vivant une situation de WFC de mieux gérer ce stress? Bien que cela ne paraisse pas faire partie de la culture française, ne serait-il pas préférable de s'intéresser plus au critère de l'efficacité qu'à celui des heures passées au bureau? En tout état de cause, l'étude de ce problème permettrait certainement d'identifier des pistes de réponses que les départements GRH des organisations pourraient proposer aux cadres vivant des situations de conflits entre leur travail et leur vie personnelle : temps partiel, congé maternité, développement d'une culture et d'un esprit ouvert aux contraintes familiales au sein même de l'organisation, etc.

\section{3 - Le rôle du droit face à une situation d'inégalité}

Depuis sa création, la Communauté Européenne a élaboré un ensemble de dispositions juridiques visant à garantir la non-discrimination à l'égard des femmes, en ce qui concerne l'accès à l'emploi, la formation professionnelle, les conditions de travail, etc. Et depuis les années 80, les programmes d'action sur l'égalité des chances entre les femmes et les hommes font l'objet d'un rapport annuel. Le Livre blanc de la Commission : « la politique sociale européenne - Une voie à suivre pour l'Union » (1994) a pour but de passer en revue les progrès réalisés en matière d'égalité au niveau des Etats membres. En France, le 7 mars 2000, l'Assemblée Nationale a débattu d'une proposition de loi sur l'égalité professionnelle. Droite et gauche ont affirmé leur désir de faire craquer le «plafond de verre » qui écarte les femmes des postes de responsabilité et les pénalise dans leur rémunération. Cette proposition devrait « compléter de façon positive » la loi Roudy de 1983 sur l'égalité professionnelle. A travers ces constats, une chose est certaine, en Europe les gouvernements ont bien conscience de l'existence de l'inégalité professionnelle entre hommes et femmes.

Cependant, les échecs de ce genre de loi, ou les bien maigres résultats atteints en comparaison aux ambitions de ces programmes, montrent bien que la loi et son utilisation ne sont pas des démarches triviales dans ce genre de phénomène.

Soulignons qu'en matière de non discrimination, au niveau du politique, on peut dénombrer deux attitudes : une passive et une active. L'attitude passive est une politique de non discrimination qui se contenterait d'interdire aux employeurs toute pratique discriminatoire dans le recrutement, l'emploi et la promotion des travailleurs. L'attitude active, elle, inciterait les employeurs à recruter du personnel au sein de certains groupes sociaux considérés comme défavorisés (Stephen, 1995).

La question pourrait être : quelle forme de non-discrimination est-il judicieux d'adopter? Active ou passive ? De simples mesures incitatives passives ou une réelle politique de préférence active?

Même si tout le monde est d'accord sur le fait que la bonne volonté ne suffit pas pour assurer une meilleure représentation des minorités, sur la question de légiférer et de contrôler, les avis ne peuvent que diverger.

Dans l'attitude active, certains juristes remettent en cause la légitimité constitutionnelle des mesures qui vont, selon eux, à l'encontre de l'objectif visé : une société où serait abolie toute discrimination, dans un sens ou l'autre. Certaines femmes aussi dénoncent les 
mesures « paternalistes », qui maintiennent les femmes dans un statut d'assistées. Beaucoup d'arguments juridiques, économiques, éthiques ou simplement pragmatiques, ramènent le problème à une question de mérite et de favoritisme, de droit des individus et de droit des groupes, ou encore à une question d'acceptation ou de négation de la différence (Steinberg, 1996). Questions qui, il faut le souligner, dépassent et de loin, le champ de la gestion, et ne facilitent pas les débats. En tout cas, sur un tel sujet, même si parfois « il est plus facile de légaliser certaines choses que de les légitimer» (Chamfort, Maximes et Pensées), la loi et son maniement ne peuvent susciter que débats et réflexions.

\section{Conclusion}

Commençons, en guise de conclusion, par rappeler, au risque de paraître redondant, que le thème des femmes cadres et leur difficulté d'accès à des postes de haut management demeure quasiment absent des préoccupations des chercheurs en gestion français. Et pourtant, comme nous avons tenté de le souligner, l'importance de son intérêt n'est pas des moindres.

En fait, le passage à l'intérêt managérial que propose ce thème n'est pas encore très bien assimilé par tous. La problématique, aux Etats-Unis par exemple, s'est presque détachée du champ social pour n'appartenir désormais qu' au champ de la gestion. Certes, lorsqu'on s'attelle à un sujet portant sur les femmes cadres, et que l'on part de constats d'inégalité et de disparités, il est assez délicat de ne pas être perçu comme adoptant un ton militant. Délicat mais pas impossible...

Il nous semble qu'en France, le temps est venu de mener une réflexion constructive sur les femmes cadres laquelle irait dans le sens de l'intérêt général, et en particulier, dans l'intérêt des organisations, sans adopter d'attitude partisane mais en se positionnant d'un point de vue gestionnaire et parfaitement pragmatique.

C'est en tout cas le sens du message que nous espérons avoir réussi à faire passer à travers nos propos. Gageons, à l'aube d'un nouveau millénaire, que de nombreux programmes de recherche sur ce thème émergeront enfin en sciences de gestion.

\section{Bibliographie}

Adler N.J. and Izraeli D.N., "Where in the world are the women executives ? », Publication: Business Quarterly, vol. 59, n 1, 1994, pp. 89-94.

Adler N.J., « Competitive Frontiers: Women Managers in the Triade », International Studies in Management and Organisation, vol. 23, $\mathrm{n}^{\circ}$ 2, 1993, pp. 3-23.

AFFDU (1998), « Congrès de Caen », DIPLOMEES, revue trimestrielle $\mathrm{n}^{\circ} 185$, juin 1998.

AFFDU, « Séminaires AFFDU- CURRIER, 25-26 mars 1998 », DIPLOMEES, revue trimestrielle $\mathrm{n}^{\circ} 186$, septembre 1998.

Anker R., « Ségrégation professionnelle hommesfemmes : les théories en présence », Revue Internationale du Travail, vol. 136, n 3, 1997, pp. 343-369.

Bachelot R. et G. Fraisse, Deux femmes au royaume des hommes, Hachette Littérature, 1999.

Belle F., « Cadres au féminin : de la revendication d'égalité à la recherche d'identité », Economie et Sociétés : Sciences de Gestion, 13, 1989, pp. 113-137.

Bhatnagar D. and R. Swamy, Attitudes toward women as managers: Does interaction make a difference?, Human Relations, Vol. 48, $\mathrm{n}^{\circ} 11,1995$, pp. 1285-1307. Bourdieu P., La domination masculine, Paris, Ed. Seuil, 1998.

Burke M., Valeurs féminines, le pouvoir de demain, Paris, Ed. Village Mondial, 1998.

Burke R.J. and Mckeen C.A., « Do women at the top make a difference ? Gender proportions and experiences of managerial and professional women », Human Relations, vol. 49, n 8, 1996, pp.1093-1104.

Burke R.J. and Davidson M., Women in Management : Current Research Issues, Paul Chapman Publishin, 1994. Conseil d'analyse économique, Egalité entre femmes et hommes : aspects économiques, rapport du Conseil d'analyse économique (présenté par Béatrice Majnoni d'Intignano), 1999. 
Coutrot T., L'entreprise néo-libérale, nouvelle utopie capitaliste?, La Découverte, 1996.

Dreher G.H. and Ash R.A., « A Comparative Study of Mentoring Among Men and Women in Managerial, Professional, and Technical Position », Journal of Applied Psychology, 75, 1990, pp. 539-546.

Duchéneaut B., «Le management féminin : émergence, spécificités et perspectives », Gestion 2000, mai-juin 1999, pp. 97-115.

Ely R J., « The power in demography : women's social constructions of gender identity at work », Academic Management Journal, vol. 38, n $^{\circ}$ 3, 1995, pp. 589-634. Fagensen E.A., « The mentor advantage: Perceived career/job experiences of protégés vs. non-protégés », Journal of Organizational Behavior, 10, 1989, pp. 309-320. Génisson C., Egalité professionnelle hommes-femmes : davantage de mixité professionnelle pour plus d'égalité, Rapport remis au Premier Ministre, septembre 1999, 67 pages.

Greenhaus J.H. and Beutell N.J., « Sources of conflict between work and family roles ", Academy of Management Review, 10, 1985, p. 76-88.

Higgins C.A. and Duxbury L.E., « Work-family conflict: A comparison of dual-career and traditional-career men », Journal of Organizations Behavior, vol. 13, 1992, pp. 389-411.

Hofstede G. et Bollinger D., Les différences culturelles dans le management : comment chaque pays gère-t-il ses hommes?, Les Editions D’Organisation, 1987.

Hunt D.M. and Michael C., « Mentorship: A career training and development tool », Academy of Management Review, 8, 1983, pp. 475-485.

INSEE, enquête emploi de mars 1998.

Kanter R.M., Men and women of the corporation, Basic Books, New York, 1977.

Klauss R., « Formalized mentor relationships for management and development programs in federal government », Public Administration Review, JulyAugust 1981, pp. 489-496.

Kram K.E. and Bragar M.C., « Development Through Mentoring : A Strategic Approach », in D. Montross and C. Shinkman, eds., Carreer Development: Theory and Practice, Chicago: Thomas Press, 1992, pp. 221-254.
Kram K.E., Mentoring at work: Developmental relationships in organization life. Glenview, IL: Scott, Foresman, 1985.

Laufer J. et Fouquet A., « Les femmes dans l'entreprise : le plafond de verre est toujours là », Revue Française de Gestion, n 119 , juin- juillet-août 1998, pp. 143-144.

Laufer J. (1982), La féminité neutralisée? Les femmes cadres dans l'entreprise, Flammarion, Paris.

Le Courrier de l'UNESCO, Femmes la moitié du ciel, septembre 1995.

Le Courrier de l'UNESCO, D'où vient le racisme, mars 1996.

Maruani M., Les nouvelles frontières de l'inégalité, La découverte, 1998.

Ministère de l'emploi - 1, «Emploi et salaires : les inégalités entre femmes et hommes en mars 1998 », Premières informations et premières synthèses, Ministère de l'emploi et de la solidarité, $\mathrm{n}^{\circ}$ 32.2-99.08, 1999, pp. 1-8.

Ministère de l'emploi - 2, «Hommes et femmes au travail : des satisfactions comparables en dépit de situations inégales », Premières informations et premières synthèses, Ministère de l'emploi et de la solidarité, $\mathrm{n}^{\circ}$ 35.2-99.09, 1999, pp. 1-8.

Murray M. and Owen M., Beyond the Myths and Magic of Mentoring, San Francisco: Jossey-Bass Inc., 1991.

Noe R.A., "Women and mentoring: A review and research agenda », Academy of Management Review, 13, pp. 65-78, 1988.

Ollenburger J C. and Moore H A, A sociology of women: the intersection of patriarchy, capitalism \& colonization, New Jersey, Prentice Hall, Englewood cliffs, 1992.

Parker V.A. and Kram K.E., « Women mentoring men: Creating conditions for connection », Business Horizons, 36, 2, 1993, pp. 42-51.

Pigeyre F., « Vers une gestion des ressources humaines sexuée ? », Revue Française de Gestion, novembredécembre, 1999, 47-55.

Pigeyre F., « Entre autonomie et contrôle : les trajectoires d'accès des femmes aux positions de pouvoir », Actes $d u$ $10^{e}$ congrès de l'AGRH, Lyon, 1999, pp. 827-839.

Ragins B.R, Towsend B. and Mattis M., « Gender Gap in The ExecutiveSuite: CEO's and female Executive 
report on Breaking the Glass Ceiling », Academy of Management Executive, vol. 12, $\mathrm{n}^{\circ} 1,1998$, pp. 28-42. Ragins B.R. and Scandura T.A., « Gender differences in expected outcomes of mentoring relationships », Academy of Management Journal, 37, 4, 1994, pp. 957971.

Roche G.R., « Much ado about mentors », Harvard Business Review, 57, 1, 1979, pp. 14-31.

Scandura A., « Mentorship and career mobility: An empirical investigation », Journal of Organizational Behavior, 13, 1992, pp.169-174.

Schwartz F.N., «Women as a Business Imperative », Harvard Business Review, March-April 1992, 1992 pp. 105-113.

Stewart J., «Understunding women in organizations : toward a reconstruction of organizational theory », Administrative Science Quarterly, vol. 23(2), 336-350.
Tharenou P., Latimer S. and Conroy D. (1994), « How do you make it to the top ? an examination of influences on women's and men's managerial advancement », Academy of Management Journal, vol. 37, $\mathrm{n}^{\circ}$ 4, pp. 899-931.

Tomlinson F., Brockbank A. and Traves J., " The 'Feminization' of management? Issues of 'Sameness' and 'Difference' in the Roles and Experiences of Female and Male Retail Managers ", Gender, Work and Organization, vol. 4, n 4, October 1997, pp. 218-229. Whitely W., Dougherty T.W., and Dreher G.F., «Relationship of Career Mentoring and Socioeconomic Origin to Managers' and Professionals' early career progress », Academy of Management Journal, vol. 34, $\mathrm{n}^{\circ} 2,1991, \mathrm{pp} .331-351$.

Wirth L., "Postes de direction : les femmes vont-elles briser le "plafond de verre" ? », Revue Internationale du Travail, vol. 136, $\mathrm{n}^{\circ} 3,1997$, pp. 104-115.
Immédiatement disponible, toute la collection réimprimée de clireation of Gestion

Collection reliée : Volumes 1 à 30 , 1965 à 1995 inclus :

$25610 \mathrm{~F}$
Séparément :

Volumes 1 à 8, 1965 à 1972 relié par volume

$1180 \mathrm{~F}$
Volumes 9 à 20 , 1973 à 1984 relié par volume

\section{$1050 \mathrm{~F}$}

\section{sous forme de volumes reliés. année par année.}

Volumes 21/22, $1985 / 1986$ reliés ensemble

\section{$1245 \mathrm{~F}$}

Volume 26/27, 1991/92 reliés ensemble, $1245 \mathrm{~F}$
Volume 28 à 30, 1992/93 reliépar volume,

$1050 \mathrm{~F}$
Catalogue complet sur demande d'autres revues en langue française, que nous avons réimprimés :

Schmidt Periodicals GmbH - Roemerring 12-14

D-83075 Bad Feilnbach, Allemagne

Tél. 0049-8064-221 - Fax 0049-8064-557 